cf 1.5\%). (Verity CM, Greenwood R, Golding J. Long-term intellectual and behavioral outcomes of children with febrile convulsions. $\mathrm{N}$ Engl I Med June 11, 1998;338:1723-1728). (Reprints: Dr Verity, Child Development Centre, Box 107, Addenbrooke's Hospital, Cambridge CB2 2QQ UK).

COMMENT. Febrile convulsions do not impair academic progress, intellect, and behavior in patients followed and tested at 10 years of age. Children with complex febrile seizures have the same cognitive and behavioral outcome as those with simple febrile seizures. These UK results are similar to those of the prospective US National Collaborative Perinatal Project that found similar IQ levels in FC and seizure-free siblings at 7 year follow-up. They differ from studies showing associations between FCs and attention deficit hyperactivity disorders and learning disabilities.

\title{
ATYPICAL BENIGN EPILEPSY WITH CENTROTEMPORAL SPIKES
}

Six children treated with carbamazepine or valproate for benign epilepsy with centrotemporal spikes (BECT), and followed at the Hospital de Cruces, Vizcaya, Spain, had an atypical and deteriorating course during therapy and a rapid remission when antiepileptic drugs were discontinued. Patient 1 had a first nocturnal, partial oromotor seizure at age 6 years, The father had BECT in childhood. EEG showed the typical bilateral midtemporal spikes. After 2 weeks of carbamazepine therapy, seizures recurred several times a day and the EEG abnormality became generalized, with continuous spike-and-wave during slow wave sleep (CSWS). Clinically, he developed a receptive language disorder, dysarthria, drooling, and myoclonic jerks of perioral muscles. Carbamazepine was discontinued and phenytoin and clobazam substituted. Within one week, the language disorder and seizures subsided, and subsequent EEGs were either normal or showed only occasional midtemporal spikes. AEDs were discontinued at 10 years of age without seizure recurrence, and school performance was normal at age 15 year follow-up. Patient 2 developed Landau-Kleffner syndrome and behavior disturbance during treatment with CBZ, with recovery after drug withdrawal. Patient 3 suffered seizure recurrences and language disorder with $\mathrm{CBZ}$, and recovered when primidone was substituted. Patient 4 had severe language deterioration, seizures and CSWS on CBZ, and normalized when clobazam was substituted. Patient 5 had seizure recurrences and CSWS during valproate therapy, worsening with increased dosage, and recovery with drug withdrawal. Patient 6 developed dementia and CSWS after 6 months therapy with CBZ and VPA, and recovered when clobazam was substituted. A combination of AED effects and the syndrome itself could be responsible for the reversible epileptic and neuropsychological deterioration observed in these cases. (Prats JM, Garaizar C, Psy MLG-N, Madoz P. Antiepileptic drugs and atypical evolution of idiopathic partial epilepsy. Pediatr Neurol May 1998;18:402-406). (Respond: Dr Jose M Prats, Division of Pediatric Neurology, Department of Pediatrics, Hospital de Cruces, 48903 Baracaldo, Vizcaya, Spain).

COMMENT. Carbamazepine-induced seizure exacerbation and valproateinduced dementia in children with BECT have been reported previously. The AEDassociated worsening of symptoms and course of this epileptic syndrome is correlated with a diffuse spread of the EEG sleep abnormality, termed "continuous spike-and-wave during slow wave sleep" (CSWS). The above report emphasizes the risks involved in treating benign idiopathic partial epilepsies with carbamazepine or sodium valproate. The possible neuropsychological deterioration, with severe language disorders, including Landau-Kleffner 
syndrome, should be reason to withhold treatment when seizures are infrequent. It is noteworthy that phenytoin and clobazam treatment of BECT was successful and unattended by seizure exacerbation and language deterioration.

\section{SUDDEN DEATH IN EPILEPSY AND OCCULT CARDIAC DISEASE}

Pathological evaluations of the hearts of 7 patients with epilepsy, aged 12 to 44 years, who died suddenly are reported from the Department of Neurosciences, New Jersey Medical School, Newark. Cardiac abnormalities were found in 5 epilepsy patients and in none of the hearts of a comparison non-epileptic group. Lesions were subendocardial, mainly irreversible perivascular and interstitial fibrosis, and reversible myocyte vacuolization. Seizures had been generalized and not more than 1 per month in the year prior to death. No patient had status epilepticus. Only 2 had therapeutic levels of AEDs at postmortem. Asphyxia was not the cause of death, and only 1 had neuropathological findings, a communicating hydrocephalus. Cardiac pathology was attributed to recurrent seizures and resulting coronary vasospasm with ischemia. (Natelson BH, Suarez RV, Terrence CF, Turizo R. Patients with epilepsy who die suddenly have cardiac disease. Arch Neurol June 1998;55:857-860). (Reprints: Benjamin H Natelson MD, Department of Neurosciences, New Jersey Medical School, 88 Ross St, East Orange, NJ 07018).

COMMENT. Patients with epilepsy who die suddenly without apparent cause are likely to have a cardiac abnormality, causally related to prior recurrent seizures and vasospasm. Risk factors for sudden death in epilepsy include young age, especially African-American, ambulatory, a long history of infrequent seizures, predominantly generalized tonic-clonic, and subtherapeutic levels of AEDs.

Adolescents with a first tonic-clonic seizure and a normal EEG are candidates for a cardiac consultation and work-up to exclude a primary cardiac arrythmia, especially those involved in sports activities. The above study indicates that young patients with epilepsy may also require cardiac supervision to monitor possible coronary ischemic complications related to recurrent seizure activity. The necessity to maintain adequate AED levels in young, ambulatory and especially sports-orientated epilepsy patients is emphasized by these findings.

\section{LEARNING DISORDERS}

\section{MEDIAL TEMPORAL LOBE ACTIVATION IN LEARNING}

Regional cerebral blood flow was examined by PET recordings during multiple-trial learning in healthy volunteers tested at Guy's and St Thomas's Hospitals and the MRC Cyclotron Unit, Hammersmith Hospital, London. Eight adult subjects were presented visually with a 15-word list, either novel or repeated. Incremental learning occurred with repeated word lists and was associated with PET activation in the left medial temporal area, but not the left frontal. The left medial temporal region is activated by novel word visual stimuli and also in incremental and consolidating learning of repeated stimuli. The right frontal and precuneal regions, participating in retrieval of already learned memories, are also involved in this activation. Memory function requiring effort and elaboration of stimuli involves the left frontal region. (Kopelman MD, Stevens TG, Foli S, Grasby P. PET activation of the medial temporal lobe in learning. Brain May 1998;121:875-887). (Respond: Dr Michael Kopelman, Division of Psychiatry and Psychology, UMDS-St Thomas's Hospital, London SE1 7EH, UK). 